\title{
Article \\ COVID-19 Vaccine Hesitancy and Associated Factors among Diabetes Patients: A Cross-Sectional Survey in Changzhi, Shanxi, China
}

\author{
Ying Wang ${ }^{1, \dagger}$, Lingrui Duan ${ }^{1, \dagger}{ }^{\dagger}$ Mufan Li ${ }^{1}$, Jiayu Wang ${ }^{1}$, Jianzhou Yang ${ }^{1,2, *}$, Congying Song ${ }^{3}$, Jing Li $^{4}$, \\ Jinsheng Wang ${ }^{5}$, Jiantao Jia ${ }^{6}$ and Junjie $X \mathrm{u}^{3, *}$
}

check for updates

Citation: Wang, Y.; Duan, L.; Li, M.; Wang, J.; Yang, J.; Song, C.; Li, J.; Wang, J.; Jia, J.; Xu, J. COVID-19 Vaccine Hesitancy and Associated Factors among Diabetes Patients: A Cross-Sectional Survey in Changzhi, Shanxi, China. Vaccines 2022, 10, 129. https://doi.org/10.3390/ vaccines10010129

Academic Editor: Zixin Wang

Received: 5 December 2021

Accepted: 12 January 2022

Published: 17 January 2022

Publisher's Note: MDPI stays neutral with regard to jurisdictional claims in published maps and institutional affiliations.

Copyright: () 2022 by the authors Licensee MDPI, Basel, Switzerland. This article is an open access article distributed under the terms and conditions of the Creative Commons Attribution (CC BY) license (https:// creativecommons.org/licenses/by/ $4.0 /)$.
1 School of Epidemiology and Public Health, Shanxi Medical University, Taiyuan 032000, China; wangying@b.sxmu.edu.cn (Y.W.); dlr0875@b.sxmu.edu.cn (L.D.); lmf98@b.sxmu.edu.cn (M.L.); JiaYu12@b.sxmu.edu.cn (J.W.)

2 Department of Preventive Medicine, Changzhi Medical College, Changzhi 046000, China

3 Clinical Research Academy, Peking University Shenzhen Hospital, Peking University, Shenzhen 518000, China; songcongying@pkuszh.com

4 Renal Division, Peking University Shenzhen Hospital, Peking University, Shenzhen 518000, China; lijingp0677@pkuszh.com

5 Department of Pathology and Basic Medicine, Changzhi Medical College, Changzhi 046000, China; jshwang@czmc.edu.cn

6 Department of Pathophysiology and Basic Medicine, Changzhi Medical College, Changzhi 046000, China; jiajt985@czmc.edu.cn

* Correspondence: jianzhouyang@czmc.edu.cn (J.Y.); xujunjie@pkuszh.com (J.X.); Tel.: +86-355-315-1579 (J.Y.); +86-755-83923333-6646 (J.X.)

+ These authors contributed equally to this work.

\begin{abstract}
Patients with diabetes are more susceptible to severe acute respiratory syndrome-associated coronavirus (SARS-CoV)-2 infection, but vaccine hesitancy is a problem in this population. We investigated the prevalence of SARS-CoV-2 vaccine hesitancy among diabetes patients in China through a cross-sectional survey from April and August 2021 using a questionnaire administered to patients at two hospitals affiliated with Changzhi Medical College (Shanxi, China). The health belief model (HBM) is used examining factors influencing vaccine hesitancy. After adjusting for potential confounders, a multivariate logistic regression model was used to analyze correlations between vaccine hesitancy and associated factors. Of the 483 participants, 56.4\% (273/483) had vaccine hesitancy, including 58.2\% (159/273) who were unsure of being vaccinated and $41.8 \%(114 / 273)$ who were unwilling. Although patients considered SARS-CoV-2 infection to be serious (adjusted odds ratio $[\mathrm{aOR}]=3.90,95 \%$ confidence interval $[\mathrm{CI}]: 2.36-6.42 ; p<0.001)$, they had concerns about vaccine safety $(\mathrm{aOR}=3.05,95 \% \mathrm{CI}: 1.89-4.91 ; p<0.001)$. Relatives' vaccination status did not influence participants' willingness to be vaccinated (aOR $=2.43,95 \% \mathrm{CI}: 1.39-4.25 ; p<0.001)$. Disagreement with physicians' view that vaccination can reduce SARS-CoV-2 infection risk was independently correlated with vaccine hesitancy $(\mathrm{aOR}=2.25,95 \% \mathrm{CI}: 1.28-3.95 ; p<0.001)$. Diabetes patients in China need to be educated on SARS-CoV-2 vaccine safety and protective effects to increase the vaccination rate in this population.
\end{abstract}

Keywords: diabetes; SARS-CoV-2 vaccine; health belief model; vaccine hesitancy

\section{Introduction}

Patients with diabetes and associated complications are more susceptible to infection with severe acute respiratory syndrome-associated coronavirus (SARS-CoV-2), the causative agent of coronavirus disease 2019 (COVID-19), and have a high risk of severe COVID-19associated morbidity and mortality [1-3]. Data from the Centers for Disease Control and Prevention indicate that diabetic patients have 5- and 3-fold higher risks of contracting SARS-CoV-2 and dying from COVID-19, respectively, compared to individuals without 
diabetes [4-6]. This may be due to acute or chronic complications of diabetes that impact immunity [7-9]. Diabetes is among the fastest growing global health emergencies of the 21 st century, with an estimated 643 million people (11.3\% of the population) living with diabetes by 2030 . China holds the highest prevalence of diabetes in the world, and it is estimated that there will be 14.086 million diabetic patients in 2021 [10]. While diabetic patients are more susceptible to infection with severe acute respiratory SARS-CoV-2, there are few reports about COVID-19 vaccination safety and immunological reactions, and there is no report about the willingness of COVID-19 vaccination in the diabetic group. Despite doctors' recommendations, more proportion of the people with diabetes is reluctant to get vaccinated, compared with the general population (14.2-29.0\% vs. 8.7\%) [11-14]. As the number of people suffering from diabetes continues to increase, it will bring a huge burden to China's health and economy. Despite studies indicating increased hesitation to SARS-CoV-2 immunization in chronic illness groups, diabetic individuals are an essential component and typical of the chronic disease community [15]. However, there are various kinds of chronic patients, and it is extremely difficult to recruit all these kinds of patients in one study. Therefore, we conducted this study among diabetic patients to investigate their willingness to vaccinate.

The SARS-CoV-2 vaccine plays a critical role in overcoming the COVID-19 pandemic. A calculation model simulation study of SARS CoV-2 vaccine efficacy showed that the vaccine has to have an efficacy of at least $70 \%$ to prevent an epidemic and of at least $80 \%$ to largely extinguish an epidemic without any other measures [16]. Most phase 3 clinical trials of SARS-CoV-2 vaccines have not included patients with comorbidities including diabetes; as such, there is a lack of real-world evidence on vaccine side effects and protective effects in this population and the existing data are inconsistent. A study on the antibody levels of diabetic and non-diabetic individuals 3 weeks after immunization with two doses of BNT162b2 mRNA COVID-19 vaccination showed that the neutralizing antibody produced by diabetic patients is $4.42 \%$ lower than that of non-diabetic patients [17]. Similarly, a study from Turkey showed that diabetes patients produced lower levels of antibodies four weeks after immunization with two doses of the SARS-CoV-2 vaccine [18].

Current guidelines or recommendations for SARS-CoV-2 vaccination in diabetes patients also vary across countries. The World Health Organization, Europe, United States, and South Korea recommend vaccination [19-24], but Chinese guidelines exclude individuals with chronic diseases that are poor-controlled by medication [25]. The lack of direct evidence on the benefits of vaccination has led vaccine hesitancy among diabetes patients, with rates of $14.2 \%$ in Italy [11], 29.0\% in Saudi Arabia [12], and 24.7\% in Malaysia [26]. Previous studies on vaccine hesitancy in patients with diabetes have focused on high-income countries, and there is limited information on prevailing attitudes in low- and middleincome countries. A recent survey conducted in Uganda found that $29.90 \%$ of patients with chronic illnesses, including diabetes, were hesitant to receive the SARS-CoV-2 vaccine [27]. The main reasons for vaccine hesitancy among diabetes patients are concerns regarding vaccination side effects and uncertainty about vaccine composition $[11,12,26]$. To date, no studies have used a theory-based analysis to investigate the reasons for vaccine hesitancy.

The Health Belief Model (HBM) is a health education model that includes information in six dimensions, including Perceived susceptibility, Perceived severity, Perceived benefits, Perceived barriers, self-efficacy to engage in a behavior, and Action clues [28]. The HBM has been used to measure population's attitudes towards to vaccination against papillomavirus (HPV), influenza, H1N1, and SARS CoV-2 virus [29-33]. The measured outcomes are also helpful to analyze behavior-related influencing factors and to change the vaccination behaviors, cognitions, attitudes, and beliefs of individuals through health education.

In China, the main vaccines used for SARS-CoV-2 are inactivated vaccines. As there are no data on the willingness of diabetes patients to receive the vaccine in China, we carried out a cross-sectional survey based on the HBM to determine the prevalence of SARS-CoV-2 vaccine hesitancy in this population, as well as the associated factors. 


\section{Materials and Methods}

\subsection{Study Design}

In this cross-sectional study conducted from April to August 2021, a questionnaire was administered through face-to-face interviews to diabetes patients hospitalized at the endocrinology department of Heping and Heji Hospitals, Changzhi Medical College (Changzhi, Shanxi, China). Information on sociodemographic characteristics, lifestyle, and disease-related conditions and pertaining to SARS-CoV-2 vaccination, such as attitudes toward vaccination and potential reasons for vaccine hesitancy, was collected.

The questionnaire was prepared by two professors of epidemiology and a clinically experienced endocrinologist after a review of the literature on the willingness to receive the SARS-CoV-2 vaccine in China and other countries. The questionnaire included three major items: (1) demographic information and health status; (2) perceived risk of SARSCoV-2 infection and knowledge of SARS-CoV-2 vaccines; and (3) willingness to receive a SARS-CoV-2 vaccine.

Demographic information, such as sex, age, ethnicity, education level, occupation, health insurance, diabetes prevalence, and family history, along with the current health status of diabetes patients, including other chronic diseases, diabetes complications, and the extent of glycemic control, were recorded.

The primary outcome of the study was participants' attitude toward future SARS-CoV-2 vaccination, with "Willing to be vaccinated", "Unwilling to be vaccinated", and "Unsure" as possible responses. Based on a previous survey [34], responses of "Unwilling to be vaccinated" and "Unsure" were classified as hesitancy to receive the SARS-CoV-2 vaccine.

We used the HBM to assess patients' hesitancy to receive the SARS-CoV-2 vaccine. The model included the following information: (1) Perceived susceptibility ("The risk of acquiring SARS CoV-2 is high"), (2) Perceived severity ("The SARS CoV-2 syndrome is severe"), (3) Perceived benefits ("Vaccination reduces the risk of infection", "Vaccination reduces the risk of transmission to other people", and "Vaccination is good for yourself and others"), (4) Perceived barriers ("As a person with diabetes, I worried about the safety of the vaccination" and "Worried about side effects of vaccination"), and (5) Action cues ("Relatives' vaccination action will affect your vaccination behavior", "Believe in the doctor's statement that vaccination can reduce the risk of infection", and "Advice on vaccination from the internet/media"). Dimensions for the above questions were as follows: (1) strongly disagree, (2) disagree, (3) neutral, (4) agree, and (5) strongly agree.

\subsection{Inclusion and Exclusion Criteria}

Eligible participants were patients diagnosed with type 1 or 2 diabetes who were hospitalized in the two participating hospitals during the study period, Age older than 18 years, and voluntarily participated in the survey and provided written, informed consent. Patients who had been diagnosed with a mental illness or had taken medication for a mental illness in the preceding 3 months, had obvious dementia symptoms, or were unable to communicate verbally with the investigators were excluded.

\subsection{Sample Size Calculation}

According to the sample size formula of estimating a total rate parameter through a cross-sectional study, $\alpha$ is the significance level and it is taken as $0.05, Z 1-\alpha / 2$ is taken as 1.96; prior to the formal survey, we derived an estimated hesitation rate for COVID-19 vaccination of $48.3 \%(29 / 60)$ in local diabetic patients through a pilot-survey; furthermore, $\delta$ is the allowable error and $\delta$ is taken as 0.05 . Therefore, the initial minimum sample size required for the study is 384 participants. In addition, based on the consideration of the participant non-response rate (10\%) and the qualified rate of questionnaire (90\%), the estimated minimum sample size was 474 . 


\subsection{Statistical Analysis}

All analyses were performed using SPSS v25.0 software (IBM, Armonk, NY, USA). Qualitative data are presented as frequencies and percentages; quantitative variables are presented as mean and standard deviation, and an independent samples t-test was used for analysis. The crude odds ratio (cOR) was obtained using univariate logistic regression model, with SARS-CoV-2 vaccine hesitancy as the dependent variable and baseline characteristics as independent variables. A multivariate analysis was performed for vaccine hesitancy, while the association between the dependent variable and independent variables of interest (i.e., those in the HBM) was assessed with adjusted dominance ratio $(\mathrm{aOR})$ and $95 \%$ confidence interval (CI). The aOR was obtained by fitting a one-way logistic regression model that included the independent variable of interest and all baseline characteristics significant at $p<0.10$.

\section{Results}

\subsection{Baseline Characteristics}

We screened 859 diabetes patients; 528 completed the survey and $45(8.5 \%)$ reported having received at least one dose of SARS-CoV-2 vaccine. Statistical analyses were conducted on 483 participants who had not received the SARS-CoV-2 vaccine. The flowchart of patient enrollment is shown in Figure 1.

Of the 483 participants, the male to female and urban to rural resident ratios were nearly 1:1. Most patients were 50-69 years old $(n=275,56.9 \%)$, Han Chinese $(n=475$, $98.3 \%)$, had less than high school education $(n=288,59.6 \%)$, were married or cohabiting with a partner $(n=424,87.8 \%)$, were farmers $(n=210,43.5 \%)$, had a per capita monthly income $<2000$ yuan $(n=205,42.4 \%)$, and urban employee insurance $(n=228,47.2 \%)$. In terms of characteristics related to lifestyles and diabetes health status, $70.0 \%(n=338)$ of the participants smoked and $74.9 \%(n=362)$ drank alcohol, $86.1 \%(n=416)$ had other chronic conditions, $67.1 \%(n=324)$ had diabetes-associated complications, $64.2 \%(n=310)$ had a family history of diabetes, and $30.2 \%(n=146)$ had a diabetes diagnosis for over 10 years. Less than $8 \%$ had a fasting glucose level $>13.9 \mathrm{mmol} / \mathrm{L}$ at the most recent testing and nearly $50 \%$ reported a postprandial glucose level $>11.1 \mathrm{mmol} / \mathrm{L}$ (Tables 1 and 2 ).

\subsection{SARS-CoV-2 Vaccination Variables for the HBM}

Regarding awareness of SARS-CoV-2, 76.8\% of the participants (371/483) did not believe that they were at high risk of SARS-CoV-2 infection, while $31.9 \%(154 / 483)$ considered COVID-19 to be a serious disease; moreover, $66.0 \%(319 / 483)$ thought that vaccination would not reduce their risk of SARS-CoV-2 infection, and 38.5\% $(186 / 483)$ thought that it would not reduce the risk of virus transmission to others. On the other hand, most patients $(95.9 \%, 463 / 483)$ thought that vaccination would be beneficial to themselves and others. Regarding perceived barriers, $69.8 \%$ of patients $(337 / 483)$ were concerned about the safety of the SARS-CoV-2 vaccine, with $58.6 \%$ (283/483) concerned about side effects. Additionally, $82.4 \%(398 / 483)$ reported that the vaccination status of relatives would not influence their vaccination behavior; moreover, $47.6 \%(230 / 483)$ disagreed with physicians' view that vaccination reduces the risk of SARS-CoV-2 infection, and 51.8\% (250/483) reported no change in their attitude toward vaccination after reading information on the internet or on social media (Table 3 ). 


\section{6 diabetic patients who were hospitalized in two affiliated hospitals of Changzhi Medical College during the project period (April to August, 2021)}

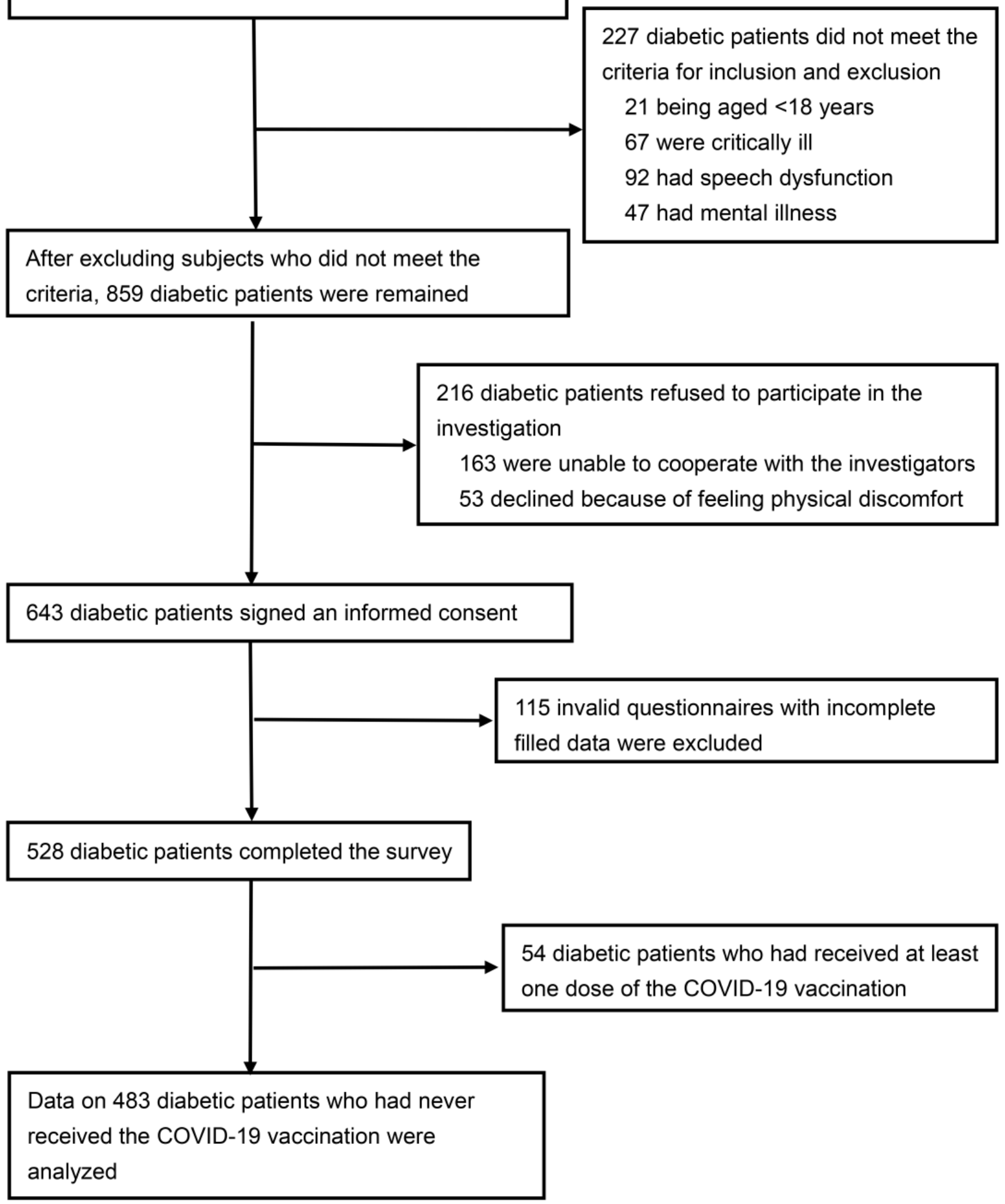

Figure 1. Flowchart of data collection. 
Table 1. Sociodemographic characteristics of the study population. $(n=483)$.

\begin{tabular}{|c|c|c|c|c|c|}
\hline Characteristic & $\begin{array}{l}\text { All Participants } \\
\begin{array}{c}(n=483) \\
\overline{\mathbf{x}} \pm \mathrm{S}\end{array}\end{array}$ & $\begin{array}{c}\text { Participants Willing to } \\
\text { Receive SARS-CoV-2 } \\
\text { Vaccine } \\
(n=210) \\
\bar{x} \pm S\end{array}$ & $\begin{array}{c}\text { Participants Hesitant to } \\
\text { Receive } \\
\text { SARS CoV-2 Vaccine } \\
(n=273) \\
\overline{\mathbf{x}} \pm \mathrm{S}\end{array}$ & $\begin{array}{l}\text { Hesitant vs. } \\
\text { Willing } \\
\text { Participants, } \\
\text { c OR/t } \\
(95 \% \mathrm{CI})\end{array}$ & $p$-Value \\
\hline \multicolumn{6}{|l|}{ Sex } \\
\hline Male & $252(52.2)$ & $114(54.3)$ & $138(50.5)$ & 1.0 & Ref \\
\hline Female & $231(47.8)$ & $96(45.7)$ & $135(49.5)$ & $0.86(0.60-1.24)$ & 0.42 \\
\hline Average age, years & $56.43 \pm 13.03$ & $56.41 \pm 12.37$ & $56.45 \pm 13.54$ & $-0.03(-2.39-2.31)$ & 0.97 \\
\hline $\begin{array}{l}\text { Age group, years } \\
18-39 \\
40-49 \\
50-59 \\
60-69 \\
\geq 70\end{array}$ & $\begin{array}{c}50(10.4) \\
78(16.1) \\
143(29.6) \\
132(27.3) \\
80(16.6)\end{array}$ & $\begin{array}{l}19(9.0) \\
43(20.5) \\
59(28.1) \\
55(26.2) \\
34(16.2)\end{array}$ & $\begin{array}{l}31(11.4) \\
35(12.8) \\
84(30.8) \\
77(28.2) \\
46(16.8)\end{array}$ & $\begin{array}{c}1.0 \\
1.21(0.56-2.49) \\
0.60(0.32-1.13) \\
1.05(0.60-1.83) \\
1.03(0.59-1.82)\end{array}$ & $\begin{array}{l}\text { Ref } \\
0.61 \\
0.11 \\
0.86 \\
0.91\end{array}$ \\
\hline \multicolumn{6}{|l|}{ Ethnicity } \\
\hline $\begin{array}{l}\text { Han } \\
\text { Other }\end{array}$ & $\begin{array}{c}475(98.3) \\
8(1.7)\end{array}$ & $\begin{array}{c}209(99.5) \\
1(0.5)\end{array}$ & $\begin{array}{l}266(97.4) \\
7(2.6)\end{array}$ & $\begin{array}{c}1.0 \\
0.18(0.02-1.49)\end{array}$ & $\begin{array}{l}\text { Ref } \\
0.11\end{array}$ \\
\hline \multicolumn{6}{|l|}{ Education level } \\
\hline $\begin{array}{l}\text { Below high school } \\
\text { High school }\end{array}$ & $\begin{array}{l}288(59.6) \\
95(19.7)\end{array}$ & $114(54.3)$ & $\begin{array}{l}174(63.7) \\
50(18.3)\end{array}$ & $\begin{array}{c}1.0 \\
1.59(1.01-2.51)\end{array}$ & $\begin{array}{l}\text { Ref } \\
0.05\end{array}$ \\
\hline College and above & $100(20.7)$ & $51(24.3)$ & 49 (17.9) & $1.16(0.66-2.03)$ & 0.61 \\
\hline \multicolumn{6}{|l|}{ Marital status } \\
\hline $\begin{array}{l}\text { Unmarried, } \\
\text { divorced, separated, } \\
\text { or widowed }\end{array}$ & $59(12.2)$ & $22(10.5)$ & $37(13.6)$ & 1.0 & Ref \\
\hline $\begin{array}{l}\text { Married or } \\
\text { cohabitating }\end{array}$ & $424(87.8)$ & $188(89.5)$ & $236(86.4)$ & $1.34(0.76-2.35)$ & 0.31 \\
\hline \multicolumn{6}{|l|}{ Occupation type } \\
\hline Farmer & $210(43.5)$ & $92(43.8)$ & $118(43.2)$ & 1.0 & Ref \\
\hline $\begin{array}{l}\text { Public institution } \\
\text { personnel }\end{array}$ & $90(18.6)$ & $49(23.3)$ & $41(15.0)$ & $0.65(0.40-1.07)$ & 0.09 \\
\hline Business staff & 55 (11.4) & $17(8.1)$ & $38(13.9)$ & $1.74(0.93-3.28)$ & 0.08 \\
\hline Worker & 74 (15.3) & $32(15.2)$ & $42(15.4)$ & $1.02(0.60-1.75)$ & 0.93 \\
\hline Transportation staff & $29(6.0)$ & $11(5.2)$ & $18(6.6)$ & $1.28(0.57-2.83)$ & 0.55 \\
\hline Other & $25(5.2)$ & $9(4.3)$ & $16(5.9)$ & $1.39(0.59-3.28)$ & 0.46 \\
\hline \multicolumn{6}{|l|}{ Residence } \\
\hline Urban & $267(55.3)$ & $115(54.8)$ & $152(55.7)$ & 1.0 & Ref \\
\hline Rural & $216(44.7)$ & 95 (45.2) & $121(44.3)$ & $1.04(0.72-1.49)$ & 0.84 \\
\hline \multicolumn{6}{|c|}{ Monthly personal income $\left(\right.$ Chinese yuan $\left.{ }^{+}\right)$} \\
\hline$<2000$ & 205 & 85 (40.5) & $120(44.0)$ & 1.0 & Ref \\
\hline $2000-3499$ & $142(29.4)$ & $58(27.6)$ & $84(30.8)$ & $0.92(0.47-1.81)$ & 0.82 \\
\hline $3500-4999$ & $93(19.3)$ & $50(23.8)$ & 43 (15.8) & $0.95(0.47-1.90)$ & 0.88 \\
\hline$\geq 5000$ & $43(8.9)$ & $17(8.1)$ & $26(9.5)$ & $0.56(0.27-1.17)$ & 0.13 \\
\hline \multicolumn{6}{|l|}{ Type of insurance } \\
\hline Útrban worker & $228(47.2)$ & $108(51.4)$ & $120(44.0)$ & 1.0 & Ref \\
\hline $\begin{array}{l}\text { Urban residents } \\
\text { New Rural }\end{array}$ & $113(23.4)$ & $41(19.5)$ & $72(26.4)$ & $0.84(0.55-1.28)$ & 0.41 \\
\hline $\begin{array}{l}\text { Cooperative Medical } \\
\text { Scheme }\end{array}$ & $142(29.4)$ & $61(29.0)$ & $81(29.7)$ & $1.32(0.80-2.20)$ & 0.28 \\
\hline
\end{tabular}

Data are shown as $\mathrm{n}(\%) .{ }^{\dagger} \mathrm{A}$ currency exchange rate of 1 Chinese yuan $=\mathrm{US} \$ 0.16$ was applied. Abbreviations: $\mathrm{CI}$, confidence interval; cOR, crude odds ratio; Ref, reference; SARS-CoV-2, severe acute respiratory syndromeassociated coronavirus 2 .

Table 2. Lifestyle and health conditions of study participants.

\begin{tabular}{|c|c|c|c|c|c|}
\hline Condition & $\begin{array}{l}\text { All Participants } \\
\begin{array}{c}(n=483) \\
\overline{\mathbf{x}} \pm \mathrm{S}\end{array}\end{array}$ & $\begin{array}{c}\text { Participants Willing to } \\
\text { Receive SARS-CoV-2 } \\
\text { Vaccine } \\
\overline{\mathbf{x}} \pm S \\
(n=210)\end{array}$ & $\begin{array}{c}\text { Participants Hesitant to } \\
\text { Receive SARS-CoV-2 } \\
\text { Vaccine } \\
\bar{x} \pm S \\
(n=273)\end{array}$ & $\begin{array}{l}\text { Hesitant vs. } \\
\text { Willing } \\
\text { Participants, } \\
\text { c OR/t } \\
(95 \% \mathrm{CI})\end{array}$ & $p$-Value \\
\hline \multicolumn{6}{|l|}{ Current smoker } \\
\hline No & $145(30.0)$ & $61(29.0)$ & $84(30.8)$ & 1.0 & Ref \\
\hline Yes & $338(70.0)$ & $149(71.0)$ & $189(69.2)$ & $1.10(0.73-1.61)$ & 0.68 \\
\hline \multicolumn{6}{|l|}{ Current drinker } \\
\hline No & $121(25.1)$ & $52(24.8)$ & $69(25.3)$ & 1.0 & Ref \\
\hline Yes & $362(74.9)$ & $158(75.2)$ & $204(74.7)$ & $1.03(0.68-1.56)$ & 0.90 \\
\hline \multicolumn{6}{|c|}{ Average self-reported BMI, $\mathrm{kg} / \mathrm{m}^{2}$} \\
\hline & $25.27 \pm 4.85$ & $25.16 \pm 5.28$ & $25.36 \pm 4.50$ & $-0.44(-1.07-0.68)$ & 0.66 \\
\hline \multicolumn{6}{|c|}{ Self-reported BMI, kg/m² } \\
\hline$<18.5^{1}$ & $55(11.4)$ & $23(11.0)$ & $32(11.7)$ & 1.0 & Ref \\
\hline $18.5-23.9$ & $186(38.5)$ & $88(41.9)$ & $98(35.9)$ & $0.86(0.43-1.73)$ & 0.68 \\
\hline $24.0-27.9$ & $161(33.3)$ & $68(32.4)$ & $93(34.1)$ & 0.69 (0.41-1.18) & 0.17 \\
\hline$\geq 28$ & $81(16.8)$ & $31(14.8)$ & $50(18.3)$ & $0.85(0.49-1.46)$ & 0.55 \\
\hline
\end{tabular}


Table 2. Cont.

\begin{tabular}{|c|c|c|c|c|c|}
\hline Condition & $\begin{array}{l}\text { All Participants } \\
\begin{array}{c}(n=483) \\
\overline{\mathbf{x}} \pm \mathrm{S}\end{array}\end{array}$ & $\begin{array}{c}\text { Participants Willing to } \\
\text { Receive SARS-CoV-2 } \\
\text { Vaccine } \\
\bar{x} \pm S \\
(n=210)\end{array}$ & $\begin{array}{c}\text { Participants Hesitant to } \\
\text { Receive SARS-CoV-2 } \\
\text { Vaccine } \\
\overline{\mathbf{x}} \pm S \\
(n=273)\end{array}$ & $\begin{array}{l}\text { Hesitant vs. } \\
\text { Willing } \\
\text { Participants, } \\
\text { c OR/t } \\
(95 \% \mathrm{CI})\end{array}$ & $p$-Value \\
\hline \multicolumn{6}{|c|}{ Other chronic diseases } \\
\hline No & $67(13.9)$ & $35(16.7)$ & $32(11.7)$ & 1.0 & Ref \\
\hline Yes & $416(86.1)$ & 175 (83.3) & $241(88.3)$ & $0.66(0.40-1.11)$ & 0.12 \\
\hline \multicolumn{6}{|c|}{ Diabetes complications } \\
\hline No & $159(32.9)$ & $73(34.8)$ & $86(31.5)$ & 1.0 & Ref \\
\hline Yes & $324(67.1)$ & $137(65.2)$ & $187(68.5)$ & $0.86(0.59-1.27)$ & 0.45 \\
\hline \multicolumn{6}{|c|}{ Controlled blood glucose level } \\
\hline No & $262(54.2)$ & 109 (51.9) & $153(56.0)$ & 1.0 & Ref \\
\hline Yes & $221(45.8)$ & $101(48.1)$ & $120(44.0)$ & $1.18(0.82-1.70)$ & 0.37 \\
\hline \multicolumn{6}{|c|}{ Family history of diabetes } \\
\hline No & $173(35.8)$ & $74(35.2)$ & $99(36.3)$ & 1.0 & Ref \\
\hline Yes & $310(64.2)$ & $136(64.8)$ & $174(63.7)$ & $1.05(0.72-1.52)$ & 0.82 \\
\hline \multicolumn{6}{|c|}{ Average time since diabetes diagnosis, years } \\
\hline 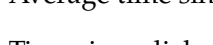 & $7.72 \pm 7.29$ & $7.58 \pm 7.49$ & $7.83 \pm 7.14$ & $-0.37(-1.57-1.07)$ & 0.71 \\
\hline \multicolumn{6}{|c|}{ Time since diabetes diagnosis, years } \\
\hline$\leq 1$ & $196(40.6)$ & $\begin{array}{l}63(30.0) \\
84(40.0)\end{array}$ & $\begin{array}{l}78(28.6) \\
112(41.0)\end{array}$ & $0.94(0.59-1.50)$ & Ref \\
\hline$>10$ & $146(30.2)$ & $63(30.0)$ & $83(30.4)$ & $1.01(0.66-1.56)$ & 0.96 \\
\hline \multicolumn{6}{|c|}{ Average fasting blood glucose at most recent testing, mmol/L } \\
\hline & $8.67 \pm 4.99$ & $8.68 \pm 6.75$ & $8.66 \pm 3.02$ & $0.04(-0.89-0.92)$ & 0.97 \\
\hline \multicolumn{6}{|c|}{ Fasting blood glucose at most recent testing, $\mathrm{mmol} / \mathrm{L}$} \\
\hline$<7$ & $169(35.0)$ & $77(36.7)$ & $92(33.7)$ & 1.0 & Ref \\
\hline$>13.9$ & $\begin{array}{c}276(57.1) \\
38(7.9)\end{array}$ & $\begin{array}{c}120(5 \% .1) \\
13(6.2)\end{array}$ & $\begin{array}{c}156(5 \% .1) \\
25(9.2)\end{array}$ & $\begin{array}{l}0.62(0.30-1.30) \\
0.68(0.33-1.38)\end{array}$ & $\begin{array}{l}0.21 \\
0.28\end{array}$ \\
\hline \multicolumn{6}{|c|}{ Average postprandial blood glucose at most recent testing, mmol/L } \\
\hline & $12.74 \pm 11.60$ & $12.32 \pm 9.81$ & $13.06 \pm 12.83$ & $-0.69(-2.84-1.36)$ & 0.49 \\
\hline \multicolumn{6}{|c|}{ Postprandial blood glucose at most recent testing, $\mathrm{mmol} / \mathrm{L}$} \\
\hline$<10$ & $159(32.9)$ & $68(32.4)$ & $91(33.3)$ & 1.0 & Ref \\
\hline $10-11.1$ & $87(18.0)$ & 46 (21.9) & $41(15.0)$ & $0.91(0.61-1.37)$ & 0.65 \\
\hline$>11.1$ & $237(49.1)$ & $96(45.7)$ & $141(51.6)$ & $0.66(0.35-1.24)$ & 0.19 \\
\hline
\end{tabular}

Data are shown as $n(\%)$. Abbreviations: BMI, body mass index; $\mathrm{CI}$, confidence interval; cOR, crude odds ratio Ref, reference; SARS-CoV-2, severe acute respiratory syndrome-associated coronavirus 2.

Table 3. Willingness to receive the SARS-CoV-2 vaccine among study participants and variables included in the health belief model.

\begin{tabular}{|c|c|c|c|c|c|}
\hline Variable & $\begin{array}{l}\text { All Participants } \\
\qquad(n=483)\end{array}$ & $\begin{array}{c}\text { Participants Willing to } \\
\text { Receive SARS-CoV-2 } \\
\text { Vaccine } \\
(n=210)\end{array}$ & $\begin{array}{c}\text { Participants Hesitant to } \\
\text { Receive SARS-CoV-2 } \\
\text { Vaccine } \\
(n=273)\end{array}$ & $\begin{array}{c}\text { Hesitant vs. } \\
\text { Willing } \\
\text { Participant, c OR } \\
(95 \% \text { CI })\end{array}$ & $p$-Value \\
\hline \multicolumn{6}{|c|}{ Willing to receive SARS-CoV-2 vaccine } \\
\hline $\begin{array}{l}\text { No (unwilling or } \\
\text { unsure) }\end{array}$ & $273(56.4)$ & $0(0)$ & $273(100)$ & $\mathrm{N} / \mathrm{A}$ & $\mathrm{N} / \mathrm{A}$ \\
\hline $\begin{array}{l}\text { Yes (willing) } \\
\text { Perceived susceptibil }\end{array}$ & $210(43.6)$ & $210(100)$ & $0(0)$ & $\mathrm{N} / \mathrm{A}$ & $\mathrm{N} / \mathrm{A}$ \\
\hline \multicolumn{6}{|c|}{$\begin{array}{l}\text { Perceived susceptibility } \\
\text { Risk of acquiring SARS-CoV-2 is high } \\
\text { No (strongly }\end{array}$} \\
\hline $\begin{array}{l}\text { disagree or disagree } \\
\text { or neutral) }\end{array}$ & $371(76.8)$ & $157(74.8)$ & $214(78.4)$ & 1.0 & Ref \\
\hline $\begin{array}{l}\text { Yes (agree or } \\
\text { strongly agree) }\end{array}$ & $112(23.2)$ & $53(25.2)$ & $59(21.6)$ & $1.22(0.80-1.87)$ & 0.35 \\
\hline \multicolumn{6}{|c|}{$\begin{array}{l}\text { SARS-CoV-2 syndrome is severe } \\
\text { No (strongly }\end{array}$} \\
\hline $\begin{array}{l}\text { disagree or disagree } \\
\text { or neutral) }\end{array}$ & $329(68.1)$ & $177(84.3)$ & $152(55.7)$ & 1.0 & Ref \\
\hline $\begin{array}{l}\text { Yes (agree or } \\
\text { strongly agree) } \\
\text { Perceived benefits }\end{array}$ & 154 (31.9) & $33(15.7)$ & $121(44.3)$ & $4.27(2.75-6.64)$ & $<0.001$ \\
\hline \multicolumn{6}{|c|}{ Vaccination reduces the risk of infection } \\
\hline $\begin{array}{l}\text { Yes (agree or } \\
\text { strongly agree) } \\
\text { No (strongly }\end{array}$ & $164(34.0)$ & $89(42.4)$ & $75(27.5)$ & 1.0 & Ref \\
\hline $\begin{array}{l}\text { disagree or disagree } \\
\text { or neutral) }\end{array}$ & $319(66.0)$ & $121(57.6)$ & $198(72.5)$ & $1.94(1.33-2.84)$ & 0.001 \\
\hline
\end{tabular}


Table 3. Cont.

\begin{tabular}{|c|c|c|c|c|c|}
\hline Variable & $\begin{array}{l}\text { All Participants } \\
\quad(n=483)\end{array}$ & $\begin{array}{c}\text { Participants Willing to } \\
\text { Receive SARS-CoV-2 } \\
\text { Vaccine } \\
(n=210)\end{array}$ & $\begin{array}{c}\text { Participants Hesitant to } \\
\text { Receive SARS-CoV-2 } \\
\text { Vaccine } \\
(n=273)\end{array}$ & $\begin{array}{c}\text { Hesitant vs. } \\
\text { Willing } \\
\text { Participant, c OR } \\
\text { (95\% CI) }\end{array}$ & $p$-Value \\
\hline \multicolumn{6}{|c|}{ Vaccination reduces the risk of transmission to other people } \\
\hline $\begin{array}{l}\text { Yes (agree or very } \\
\text { agree) }\end{array}$ & $297(61.5)$ & $149(71.0)$ & $148(54.2)$ & 1.0 & Ref \\
\hline $\begin{array}{l}\text { No (strongly } \\
\text { disagree or disagree } \\
\text { or neutral) }\end{array}$ & $186(38.5)$ & $61(29.0)$ & $125(45.8)$ & $2.06(1.41-3.02)$ & $<0.001$ \\
\hline \multicolumn{6}{|c|}{ Vaccination is good for myself and others } \\
\hline $\begin{array}{l}\text { Yes (agree or } \\
\text { strongly agree) } \\
\text { No (strongly }\end{array}$ & $463(95.9)$ & $202(96.2)$ & $261(95.6)$ & 1.0 & Ref \\
\hline $\begin{array}{l}\text { disagree or disagree } \\
\text { or neutral) }\end{array}$ & $20(4.1)$ & $8(3.8)$ & $12(4.4)$ & $1.16(0.47-2.89)$ & 0.75 \\
\hline \multicolumn{6}{|c|}{$\begin{array}{l}\text { Perceived barriers } \\
\text { As a person with diabetes, I worry about the safety of the SARS-CoV-2 vaccine } \\
\text { No (strongly }\end{array}$} \\
\hline $\begin{array}{l}\text { disagree or disagree } \\
\text { or neutral) }\end{array}$ & $146(30.2)$ & $95(45.2)$ & $51(18.7)$ & 1.0 & Ref \\
\hline $\begin{array}{r}\text { Yes (agree or } \\
\text { strongly agree) }\end{array}$ & $337(69.8)$ & $115(54.8)$ & $222(81.3)$ & $3.60(2.39-5.41)$ & $<0.001$ \\
\hline \multicolumn{6}{|c|}{$\begin{array}{l}\text { I am worried about side effects of vaccination } \\
\text { No (strongly }\end{array}$} \\
\hline $\begin{array}{l}\text { disagree or disagree } \\
\text { or neutral) }\end{array}$ & $200(41.4)$ & $116(55.2)$ & $84(30.8)$ & 1.0 & Ref \\
\hline $\begin{array}{l}\text { Yes (agree or } \\
\text { strongly agree) } \\
\text { Action clues }\end{array}$ & 283 (58.6) & $94(44.8)$ & $189(69.2)$ & $2.77(1.91-4.04)$ & $<0.001$ \\
\hline \multicolumn{6}{|c|}{$\begin{array}{l}\text { Action clues } \\
\text { Relatives' vaccination status will affect my vaccination behavior }\end{array}$} \\
\hline $\begin{array}{l}\text { Yes (agree or } \\
\text { strongly agree) } \\
\text { No (strongly }\end{array}$ & $85(17.6)$ & $52(24.8)$ & $33(12.1)$ & 1.0 & Ref \\
\hline $\begin{array}{l}\text { disagree or disagree } \\
\text { or neutral) }\end{array}$ & $398(82.4)$ & $158(75.2)$ & $240(87.9)$ & $2.40(1.48-3.87)$ & $<0.001$ \\
\hline \multicolumn{6}{|c|}{ I accept physicians' view that vaccination can reduce the risk of infection } \\
\hline $\begin{array}{l}\text { Yes (agree or } \\
\text { strongly agree) } \\
\text { No (strongly }\end{array}$ & $253(52.4)$ & $150(71.4)$ & $103(37.7)$ & 1.0 & Ref \\
\hline $\begin{array}{l}\text { disagree or disagree } \\
\text { or neutral) }\end{array}$ & $230(47.6)$ & $60(28.6)$ & $170(62.3)$ & $4.13(2.80-6.07)$ & $<0.001$ \\
\hline \multicolumn{6}{|c|}{ I have received advice on vaccination from the internet/media } \\
\hline $\begin{array}{l}\text { Yes (agree or } \\
\text { strongly agree) } \\
\text { No (strongly }\end{array}$ & $233(48.2)$ & $124(59.0)$ & $109(39.9)$ & 1.0 & Ref \\
\hline $\begin{array}{l}\text { disagree or disagree } \\
\text { or neutral) }\end{array}$ & $250(51.8)$ & $86(41.0)$ & $164(60.1)$ & $2.17(1.50-3.13)$ & $<0.001$ \\
\hline
\end{tabular}

Data are shown as $\mathrm{n}(\%)$. Abbreviations: CI, confidence interval; cOR, crude odds ratio; N/A, not applicable; Ref, reference; SARS-CoV-2, severe acute respiratory syndrome-associated coronavirus 2.

\subsection{SARS-CoV-2 Vaccine Hesitancy and Associated Factors}

Less than half of the participants $(43.6 \%, 210 / 483)$ were willing to receive the SARSCoV-2 vaccine; among the $56.4 \%(273 / 483)$ who were reluctant to be vaccinated, $58.2 \%$ $(159 / 273)$ were unsure of whether to be vaccinated and $41.8 \%(114 / 273)$ were unwilling to be vaccinated in the future.

In the univariate logistic regression analysis, willingness to receive the SARS-CoV-2 vaccine was lower among diabetes patients with a high school education level. Lifestyle factors such as smoking and health status were unrelated to vaccine hesitancy (Tables 1 and 2).

After adjusting for differences in baseline characteristics related to social context-, lifestyle-, and disease-related conditions, the perception of COVID-19 as a severe disease $(\mathrm{aOR}=3.90,95 \% \mathrm{CI}: 2.36-6.42 ; p<0.001)$, concerns regarding the safety of the SARS-CoV-2 vaccine $(\mathrm{aOR}=3.05,95 \% \mathrm{CI}: 1.89-4.91 ; p<0.001)$, disagreement with the statement that relatives' vaccination status would influence participants' vaccination decision $(\mathrm{aOR}=2.43$, 95\% CI: $1.39-4.25 ; p<0.001$ ), and disagreement with physicians' view that vaccination reduces the risk of SARS-CoV-2 infection $(\mathrm{aOR}=2.25,95 \% \mathrm{CI}: 1.28-3.95 ; p<0.001)$ were associated with vaccine hesitancy (Table 4). 
Table 4. Multivariate model of the factors associated with diabetes patients' hesitation to receive the SARS-CoV-2 vaccine.

\begin{tabular}{|c|c|c|}
\hline Variable & a OR $(95 \% C I) \dagger$ & $p$-Value \\
\hline \multicolumn{3}{|l|}{ Perceived susceptibility } \\
\hline \multicolumn{3}{|l|}{ Risk of acquiring SARS-CoV-2 is high } \\
\hline No (strongly disagree or disagree or neutral) & 1.0 & Ref \\
\hline Yes (agree or strongly agree) & $0.79(0.47-1.32)$ & 0.36 \\
\hline \multicolumn{3}{|l|}{ Perceived severity } \\
\hline \multicolumn{3}{|l|}{ SARS-CoV-2 syndrome is severe } \\
\hline No (strongly disagree or disagree or neutral) & 1.0 & Ref \\
\hline Yes (agree or strongly agree) & $3.90(2.36-6.42)$ & $<0.001$ \\
\hline \multicolumn{3}{|l|}{ Perceived benefits } \\
\hline \multicolumn{3}{|l|}{ Vaccination reduces the risk of infection } \\
\hline Yes (agree or strongly agree) & 1.0 & Ref \\
\hline No (strongly disagree or disagree or neutral) & $0.65(0.41-1.03)$ & 0.07 \\
\hline \multicolumn{3}{|c|}{ Vaccination reduces the risk of transmission to other people } \\
\hline Yes (agree or very agree) & 1.0 & Ref \\
\hline No (strongly disagree or disagree or neutral) & $1.22(0.77-1.94)$ & 0.40 \\
\hline \multicolumn{3}{|l|}{ Vaccination is good for myself and others } \\
\hline Yes (agree or strongly agree) & 1.0 & Ref \\
\hline No (strongly disagree or disagree or neutral) & $1.09(0.40-2.99)$ & 0.87 \\
\hline \multicolumn{3}{|c|}{ Perceived barriers } \\
\hline \multicolumn{3}{|c|}{ As a person with diabetes, I worry about the safety of the SARS-CoV-2 vaccine } \\
\hline No (strongly disagree or disagree or neutral) & 1.0 & Ref \\
\hline Yes (agree or strongly agree) & $3.05(1.89-4.91)$ & $<0.001$ \\
\hline \multicolumn{3}{|l|}{ I am worried about side effects of vaccination } \\
\hline No (strongly disagree or disagree or neutral) & 1.0 & Ref \\
\hline Yes (agree or strongly agree) & $0.71(0.41-1.25)$ & 0.24 \\
\hline \multicolumn{3}{|c|}{ Action clues } \\
\hline \multicolumn{3}{|c|}{ Relatives' vaccination status will affect my vaccination behavior } \\
\hline Yes (agree or strongly agree) & 1.0 & Ref \\
\hline No (strongly disagree or disagree or neutral) & $2.43(1.39-4.25)$ & 0.002 \\
\hline \multicolumn{3}{|c|}{ I accept physicians' view that vaccination can reduce the risk of infection } \\
\hline Yes (agree or strongly agree) & 1.0 & Ref \\
\hline No (strongly disagree or disagree or neutral) & $2.25(1.28-3.95)$ & 0.005 \\
\hline \multicolumn{3}{|c|}{ I have received advice on vaccination from the internet/media } \\
\hline Yes (agree or strongly agree) & 1.0 & Ref \\
\hline No (strongly disagree or disagree or neutral) & $1.29(0.83-2.00)$ & 0.26 \\
\hline
\end{tabular}

${ }^{\dagger}$ aOR was obtained by fitting a single logistic regression model involving an independent variable of interest and all background variables listed in Tables 1 and 2 with $p<0.10$ in the univariate analysis. Abbreviations: aOR, adjusted odds ratio; $\mathrm{CI}$, confidence interval; Ref, reference; SARS-CoV-2, severe acute respiratory syndromeassociated coronavirus 2 .

\subsection{Reasons for Diabetes Patients' Hesitancy to Receive the SARS-CoV-2 Vaccine}

The main reasons for the reluctance to be vaccinated against SARS-CoV-2 among diabetes patients were as follows: belief that the SARS-CoV-2 vaccine is unsafe $(11.84 \%$ in males and $12.82 \%$ in females, $p=0.95)$; fear of side effects from the vaccine $(10.97 \%$ in males and $11.22 \%$ in females, $p=0.71)$; fear of other adverse reactions after vaccination $(8.26 \%$ in males and $10.73 \%$ in females, $p=0.21$ ); no current reports of follow-up after vaccination of diabetes patients $(9.25 \%$ in males and $9.74 \%$ in females, $p=0.17)$; belief that COVID-19 is not dangerous to diabetes patients' health (3.95\% in males and $4.32 \%$ in females, $p=0.94)$; and belief that vaccination does not reduce the risk of infection $(3.95 \%$ in males and $2.96 \%$ in females, $p=0.89$ ). There were no statistically significant differences between males and females in any of the above reasons (Figure 2). 


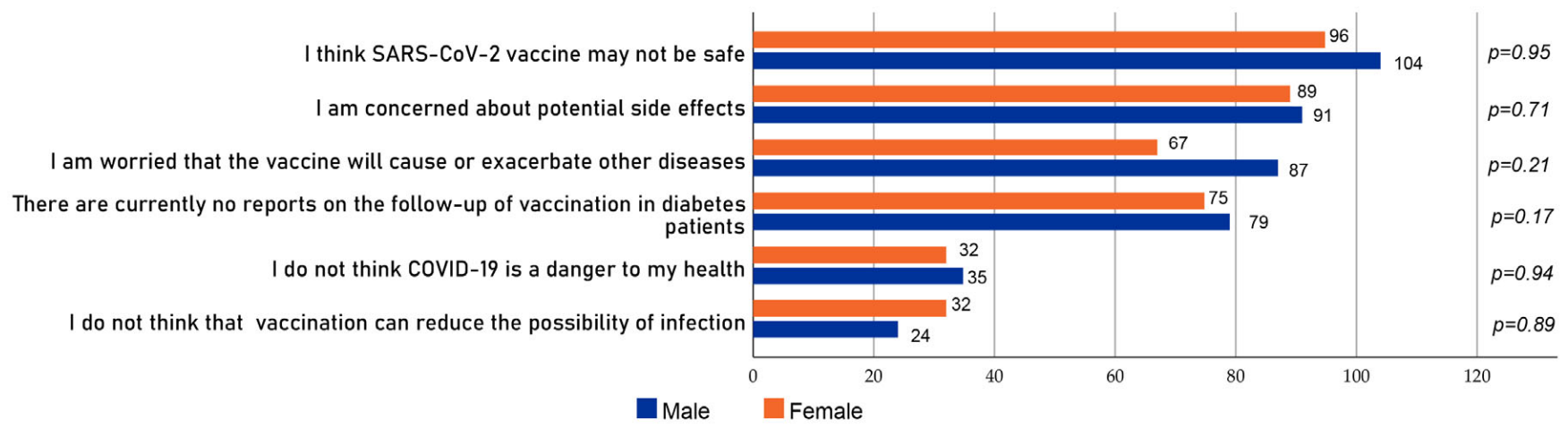

Figure 2. Reasons for SARS-CoV-2 vaccine hesitancy among study participants $(n=273)$.

\section{Discussion}

Vaccination against SARS-CoV-2 can significantly reduce the risk of COVID-19 in patients with chronic diseases including diabetes. In the published literature reports, no significant adverse effects were found in diabetics after receiving the SARS CoV-2 vaccine [35]. Therefore, initiatives to promote SARS-CoV-2 vaccination in this population are needed. The present study is the first to report the prevalence of SARS-CoV-2 vaccine hesitancy among Chinese diabetes patients. We also determined through a questionnaire based on the HBM that action cues were the main factors influencing vaccine hesitancy in our population. These findings provide a basis for targeted education and behavioral interventions to improve SARS-CoV-2 vaccine coverage in at-risk groups and reduce their risk of morbidity and mortality from COVID-19.

The proportion of patients with diabetes who were hesitant to receive the SARS-CoV-2 vaccine $(56.4 \%$, 95\%CI: 52.0-60.8) was significantly higher than in diabetes patients in higher-income countries, such as Italy (14.2-18.3\%) [11,13] and Saudi Arabia (29\%) [12], and in patients with various chronic diseases including diabetes in Uganda (29.9\%) [27] and Saudi Arabia (48\%) [36]. Thus, SARS-CoV-2 vaccine hesitancy is relatively high among Chinese diabetes patients; education and specific guidelines are needed to overcome this resistance. For example, guidelines should specify the standard of vaccination for diabetes patients based on expert consensus.

This was the first study to use the HBM and multivariate logistic regression analysis to identify factors associated with SARS-CoV-2 vaccine hesitancy among diabetes patients in China. Social contextual factors related to patients' reluctance to receive the SARS-CoV-2 vaccine included a high school education level or less. It is possible that patients with a lower education level are less likely to accept new things and were, thus, skeptical about the SARS-CoV-2 vaccine, which is supported by the results of a previous study in the general population [37]. In the future, health education initiatives should target diabetes patients with low education levels to increase the vaccination rate in this group.

Our HBM-based survey examined factors in five dimensions that could influence diabetes patients' reluctance to receive the SARS-CoV-2 vaccine. First, false beliefs regarding the risk of SARS-CoV-2 infection among diabetes patients may have led to vaccine hesitancy: most patients in our study did not think that they were at high risk of infection but agreed that the prognosis of the disease was poor for patients with diabetes, in line with a previous report [38]. Second, most patients did not believe that vaccination would reduce their risk of SARS-CoV-2 infection, but agreed that it reduced the risk of transmission and that it was, therefore, beneficial to both themselves and others. This reflects the conflicted opinion of diabetes patients, who are uncomfortable with SARS-CoV-2 vaccination because of their physical condition but believe that vaccination of other diabetes patients can reduce the risk of virus transmission. Third, we found that concerns about vaccine safety (i.e., side effects) and efficacy were barriers to patients' acceptance of the SARS-CoV-2 vaccine. This is consistent with the results of a nationwide online survey on SARS-CoV-2 vaccine hesitancy and requirement in China [31]. Fourth, the opinions and behaviors of people in their 
surroundings influenced patients' willingness to be vaccinated, including the vaccination status of relatives and physicians' view that the SARS-CoV-2 vaccine reduces the risk of infection. This is in accordance with an earlier report that diabetes patients' vaccination behavior is susceptible to the opinions of others, and would only accept vaccination if the vaccine was administered to a large group of people [31]. Fifth, the promotion of the SARS-CoV-2 vaccine on social media influenced patients' willingness to be vaccinated. It was demonstrated that receiving vaccination messages from trustworthy sources such as scientists or politicians could promote vaccine acceptance [39]. Thus, encouraging patients with diabetes to accept the SARS-CoV-2 vaccine through (social) media campaigns can increase the vaccination rate in this population [31].

This survey to the 273 diabetes patients with SARS-CoV-2 vaccine hesitancy revealed that the main reason for their reluctance was fear that the vaccine was unsafe and could have side effects. This is in line with findings from a previous study on attitudes toward influenza vaccination [40]. It is worth noting that the degree of concern among respondents was inversely related to their willingness to be vaccinated. Providing reports on the safety and side effects of the SARS-CoV-2 vaccine or firsthand accounts of patients with diabetes who have been vaccinated could allay these concerns. Moreover, the medium- and longterm effects are still unclear, and it is necessary for the health sector to further investigate the long-term immune response of diabetic patients to COVID-19 vaccination in the future, thereby improving the relevant cognition of diabetic patients as well as making more scientific decisions on vaccination behavior.

The results of this study have some practical implications. Given the factors that were identified as influencing diabetes patients' willingness to receive the SARS-CoV-2 vaccine (e.g., education level), it is possible to increase the vaccination rate in this population through education and public awareness campaigns. Additionally, interventions can be implemented to address the factors contributing to vaccine hesitancy and increase confidence in the vaccine among diabetes patients.

A strength of this study is that it is the first to investigate factors associated with SARS$\mathrm{CoV}-2$ vaccine hesitancy in diabetes patients in a low- or middle-income country using the HBM. Our findings provide a reference for promoting vaccination against SARS-CoV-2 among diabetes patients in China and other low- and middle-income countries. However, our study also had some limitations. First, the sample was not representative of all diabetes patients in China because it was limited to one city, Changzhi. Moreover, this study was only conducted in a small sample size of 483 diabetic subjects; thus, in a future study, we will recruit participants from the general population in the same period as a control group to compare results. Additionally, we did not ask patients about their history of other vaccines, which could affect their SARS-CoV-2 vaccine status. Future studies need to consider this potentially important factor. Moreover, we condensed a five-point scale (strongly disagree; disagree; neutral; agree, and strongly agree) into a binary yes/no, and thus, we were losing potential information. We should use a more complex regression to explain all five options on the ordinal scale. Lastly, as the study was a cross-sectional survey, the causal relationship between predictors and outcome variables could not be determined.

\section{Conclusions}

In conclusion, we found that Chinese patients with diabetes have a higher rate of SARS$\mathrm{CoV}-2$ vaccine hesitancy than diabetes patients in other countries. Doubt regarding vaccine efficacy, lack of awareness of the risk of SARS-CoV-2 infection, and other factors, such as SARS-CoV-2 vaccine safety (side effects) and clinicians' recommendations for SARS-CoV-2 vaccination, contribute to vaccine hesitancy. Appropriate education and interventions are needed to overcome Chinese diabetes patients' reluctance to be vaccinated and reduce the risk of COVID-19-associated morbidity and mortality in this population. 


\begin{abstract}
Author Contributions: Conceptualization, J.Y., Y.W. and L.D.; Formal analysis, Y.W. and L.D.; Writing-original draft, Y.W. and M.L.; Methodology, L.D., C.S. and J.W. (Jinsheng Wang); Data curation, J.W. (Jiayu Wang) and J.J.; Funding acquisition, J.Y.; resources, J.L.; Writing-review and editing, J.Y., J.X. and J.W. (Jinsheng Wang); Methodology Supervision, J.X. All authors have read and agreed to the published version of the manuscript.
\end{abstract}

Funding: This research was supported by the National Natural Science Foundation of China (Number 81872674) and the Academic technology leader project of Changzhi Medical College (Number XSQ201902).

Institutional Review Board Statement: The study was conducted according to the guidelines of the Declaration of Helsinki and was approved by the Institutional Review Board of Changzhi Medical College (2021065).

Informed Consent Statement: Informed consent was obtained from all subjects involved in the study. Data Availability Statement: Not applicable.

Acknowledgments: We would like to express our sincere gratitude to all the participants who enrolled in this study.

Conflicts of Interest: The authors declare no conflict of interest.

\title{
References
}

1. Huang, C.; Wang, Y.; Li, X.; Ren, L.; Zhao, J.; Hu, Y.; Zhang, L.; Fan, G.; Xu, J.; Gu, X. Clinical features of patients infected with 2019 novel coronavirus in Wuhan, China. Lancet 2020, 395, 497-506. [CrossRef]

2. Chen, N.; Zhou, M.; Dong, X.; Qu, J.; Gong, F.; Han, Y.; Qiu, Y.; Wang, J.; Liu, Y.; Wei, Y. Epidemiological and clinical characteristics of 99 cases of 2019 novel coronavirus pneumonia in Wuhan, China: A descriptive study. Lancet 2020, 395, 507-513. [CrossRef]

3. Majeed, J.; Ajmera, P.; Goyal, R.K. Delineating clinical characteristics and comorbidities among 206 COVID-19 deceased patients in India: Emerging significance of renin angiotensin system derangement. Diabetes Res. Clin. Pract. 2020, 167, 108349. [CrossRef]

4. American Diabetes Association. 4. Comprehensive Medical Evaluation and Assessment of Comorbidities: Standards of Medical Care in Diabetes-2020. Diabetes Care 2020, 43, S37-S47. [CrossRef]

5. Wang, W.; Lu, J.; Gu, W.; Zhang, Y.; Liu, J.; Ning, G. Care for diabetes with COVID-19: Advice from China. J. Diabetes 2020, 12, 417-419. [CrossRef]

6. Wu, Z.; McGoogan, J.M. Characteristics of and Important Lessons from the Coronavirus Disease 2019 (COVID-19) Outbreak in China: Summary of a Report of 72,314 Cases from the Chinese Center for Disease Control and Prevention. JAMA 2020, 323, 1239-1242. [CrossRef]

7. Vamos, E.P.; Pape, U.J.; Curcin, V.; Harris, M.J.; Valabhji, J.; Majeed, A.; Millett, C. Effectiveness of the influenza vaccine in preventing admission to hospital and death in people with type 2 diabetes. Can. Med. Assoc. J. 2016, 188, E342-E351. [CrossRef]

8. Wang, I.K.; Lin, C.L.; Chang, Y.C.; Lin, P.C.; Liang, C.C.; Liu, Y.L.; Chang, C.T.; Yen, T.H.; Huang, C.C.; Sung, F.C. Effectiveness of influenza vaccination in elderly diabetic patients: A retrospective cohort study. Vaccine 2013, 31, 718-724. [CrossRef]

9. Zhang, J.Y.; Shang, T.; Ahn, D.; Chen, K.; Coté, G.; Espinoza, J.; Mendez, C.E.; Spanakis, E.K.; Thompson, B.; Wallia, A. How to Best Protect People with Diabetes from the Impact of SARS-CoV-2: Report of the International COVID-19 and Diabetes Summit. J. Diabetes Sci. Technol. 2021, 15, 478-514. [CrossRef]

10. IDF Diabetes Atlas 10th Edition 2021. China Diabetes Report 2000-2045. Available online: https://diabetesatlas.org/data/en/ country/42/cn.html (accessed on 13 December 2021).

11. Guaraldi, F.; Montalti, M.; Di Valerio, Z.; Mannucci, E.; Nreu, B.; Monami, M.; Gori, D. Rate and Predictors of Hesitancy toward SARS-CoV-2 Vaccine among Type 2 Diabetic Patients: Results from an Italian Survey. Vaccines 2021, 9, 460. [CrossRef]

12. Aldossari, K.K.; Alharbi, M.B.; Alkahtani, S.M.; Alrowaily, T.Z.; Alshaikhi, A.M.; Twair, A.A. COVID-19 vaccine hesitancy among patients with diabetes in Saudi Arabia. Diabetes Metab. Syndr. 2021, 15, 102271. [CrossRef]

13. Scoccimarro, D.; Panichi, L.; Ragghianti, B.; Silverii, A.; Mannucci, E.; Monami, M. SARS-CoV-2 vaccine hesitancy in Italy: A survey on subjects with diabetes. Nutr. Metab. Cardiovasc. Dis. 2021, 31, 3243-3246. [CrossRef]

14. Wang, J.; Jing, R.; Lai, X.; Zhang, H.; Lyu, Y.; Knoll, M.D.; Fang, H. Acceptance of COVID-19 Vaccination during the COVID-19 Pandemic in China. Vaccines 2020, 8, 482. [CrossRef]

15. Wang, J.; Yuan, B.; Lu, X.; Liu, X.; Li, L.; Geng, S.; Zhang, H.; Lai, X.; Lyu, Y.; Feng, H. Willingness to accept COVID-19 vaccine among the elderly and the chronic disease population in China. Hum. Vaccines Immunother. 2021, 1-16. [CrossRef]

16. Bartsch, S.M.; O'Shea, K.J.; Ferguson, M.C.; Bottazzi, M.E.; Wedlock, P.T.; Strych, U.; McKinnell, J.A.; Siegmund, S.S.; Cox, S.N.; Hotez, P.J. Vaccine Efficacy Needed for a COVID-19 Coronavirus Vaccine to Prevent or Stop an Epidemic as the Sole Intervention. Am. J. Prev. Med. 2020, 59, 493-503. [CrossRef]

17. Ali, H.; Alterki, A.; Sindhu, S.; Alahmad, B.; Hammad, M.; Al-Sabah, S.; Alghounaim, M.; Jamal, M.H.; Aldei, A.; Mairza, M.J. Robust Antibody Levels in Both Diabetic and Non-Diabetic Individuals After BNT162b2 mRNA COVID-19 Vaccination. Front. Immunol. 2021, 12, 752233. [CrossRef] 
18. Karamese, M.; Tutuncu, E.E. The effectiveness of inactivated SARS-CoV-2 vaccine (CoronaVac) on antibody response in participants aged 65 years and older. J. Med. Virol. 2022, 94, 173-177. [CrossRef]

19. WHO. Interim Recommendations for Use of the ChAdOx1-S [Recombinant] Vaccine against COVID-19 (AstraZeneca COVID-19 Vaccine AZD1222 Vaxzevria ${ }^{\mathrm{TM}}$, SII COVISHIELD ${ }^{\mathrm{TM}}$ ): Interim Guidance, First Issued 10 February 2021, Updated 21 April 2021 , Last Updated 30 July 2021. Available online: https:/ /apps.who.int/iris/handle/10665/343388 (accessed on 23 October 2021).

20. European Centre for Disease Prevention and Control. COVID-19 Vaccination and Prioritisation Strategies in the EU/EEA. Available online: https://www.ecdc.europa.eu/en/publications-data/covid-19-vaccination-and-prioritisation-strategies-eueea (accessed on 2 September 2021).

21. Diabetes UK. Updates: Coronavirus and Diabetes. Available online: https://www.diabetes.org.uk/about_us/news/coronavirus (accessed on 26 October 2021).

22. Powers, A.C.; Aronoff, D.M.; Eckel, R.H. COVID-19 vaccine prioritisation for type 1 and type 2 diabetes. Lancet Diabetes Endocrinol. 2021, 9, 140-141. [CrossRef]

23. Centers for Disease Control and Prevention. The Advisory Committee on Immunization Practices' Updated Interim Recommendation for Allocation of COVID-19 Vaccine-United States, December 2020. Available online: https://www.cdc.gov/mmwr/ volumes /69/wr/mm695152e2.htm (accessed on 26 October 2021).

24. Korea Centers for Disease Control and Prevention Who First Gets the COVID-19 Vaccination? 2021. Available online: https: / / ncv.kdca.go.kr/menu.es?mid=a10117010000 (accessed on 14 August 2021).

25. National Health Commission of the People's Republic of China. New Coronavirus Vaccination Technical Guide (First Edition). Available online: https://xueshu.baidu.com/usercenter/paper/show? paperid=1a4x02c0rb0q0p00786n0mf0bj229634\&site= xueshu_se (accessed on 30 April 2021).

26. Syed Alwi, S.A.R.; Rafidah, E.; Zurraini, A.; Juslina, O.; Brohi, I.B.; Lukas, S. A survey on COVID-19 vaccine acceptance and concern among Malaysians. BMC Public Health 2021, 21, 1129. [CrossRef]

27. Bongomin, F.; Olum, R.; Andia-Biraro, I.; Nakwagala, F.N.; Hassan, K.H.; Nassozi, D.R.; Kaddumukasa, M.; Byakika-Kibwika, P.; Kiguli, S.; Kirenga, B.J. COVID-19 vaccine acceptance among high-risk populations in Uganda. Ther. Adv. Infect. Dis. 2021, 8, 20499361211024376. [CrossRef]

28. Carico, R.R., Jr.; Sheppard, J.; Thomas, C.B. Community pharmacists and communication in the time of COVID-19: Applying the health belief model. Res. Soc. Adm. Pharm. 2021, 17, 1984-1987. [CrossRef]

29. Carpenter, C.J. A meta-analysis of the effectiveness of health belief model variables in predicting behavior. Health Commun. 2010, 25, 661-669. [CrossRef]

30. Harrison, J.A.; Mullen, P.D.; Green, L.W. A meta-analysis of studies of the Health Belief Model with adults. Health Educ. Res. 1992, 7, 107-116. [CrossRef]

31. Lin, Y.; Hu, Z.; Zhao, Q.; Alias, H.; Danaee, M.; Wong, L.P. Understanding COVID-19 vaccine demand and hesitancy: A nationwide online survey in China. PLoS Negl. Trop. Dis. 2020, 14, e0008961. [CrossRef]

32. Wong, M.C.S.; Wong, E.L.Y.; Huang, J.; Cheung, A.W.L.; Law, K.; Chong, M.K.C.; Ng, R.W.Y.; Lai, C.K.C.; Boon, S.S.; Lau, J.T.F. Acceptance of the COVID-19 vaccine based on the health belief model: A population-based survey in Hong Kong. Vaccine 2021, 39, 1148-1156. [CrossRef]

33. Wong, L.P.; Alias, H.; Wong, P.F.; Lee, H.Y.; AbuBakar, S. The use of the health belief model to assess predictors of intent to receive the COVID-19 vaccine and willingness to pay. Hum. Vaccines Immunother. 2020, 16, 2204-2214. [CrossRef]

34. Fisher, K.A.; Bloomstone, S.J.; Walder, J.; Crawford, S.; Fouayzi, H.; Mazor, K.M. Attitudes Toward a Potential SARS-CoV-2 Vaccine: A Survey of U.S. Adults. Ann. Intern. Med. 2020, 173, 964-973. [CrossRef]

35. Baden, L.R.; El Sahly, H.M.; Essink, B.; Kotloff, K.; Frey, S.; Novak, R.; Diemert, D.; Spector, S.A.; Rouphael, N.; Creech, C.B. Efficacy and Safety of the mRNA-1273 SARS-CoV-2 Vaccine. N. Engl. J. Med. 2021, 384, 403-416. [CrossRef]

36. Al-Hanawi, M.K.; Ahmad, K.; Haque, R.; Keramat, S.A. Willingness to receive COVID-19 vaccination among adults with chronic diseases in the Kingdom of Saudi Arabia. J. Infect. Public Health 2021, 14, 1489-1496. [CrossRef]

37. Robinson, E.; Jones, A.; Lesser, I.; Daly, M. International estimates of intended uptake and refusal of COVID-19 vaccines: A rapid systematic review and meta-analysis of large nationally representative samples. Vaccine 2021, 39, 2024-2034. [CrossRef]

38. Bi, X.; Zhang, Q.; Fan, K.; Tang, S.; Guan, H.; Gao, X.; Cui, Y.; Ma, Y.; Wu, Q.; Hao, Y. Risk Culture and COVID-19 Protective Behaviors: A Cross-Sectional Survey of Residents in China. Front. Public Health 2021, 9, 686705. [CrossRef]

39. Al-Hasan, A.; Khuntia, J.; Yim, D. Does Seeing What Others Do Through Social Media Influence Vaccine Uptake and Help in the Herd Immunity Through Vaccination? A Cross-Sectional Analysis. Front. Public Health 2021, 9, 715931. [CrossRef]

40. Lau, A.Y.; Sintchenko, V.; Crimmins, J.; Magrabi, F.; Gallego, B.; Coiera, E. Impact of a web-based personally controlled health management system on influenza vaccination and health services utilization rates: A randomized controlled trial. J. Am. Med. Inform. Assoc. 2012, 19, 719-727. [CrossRef] 